\title{
Die Bestimmung des Kaliums als Kaliumplatinchlorid.
}

\author{
Von
}

\section{Dr. H. Precht.}

Von den verschiedenen, in den Lehrbüchern der analytischen Chemie vorgeschlagenen Kaliumbestimmungsmethoden ist wohl nur diejenige mittelst Platinchlorids zur allgemeinen Anwendung gekommen. Alle übrigen Methoden sind weniger zuverlässig und haben sich in der Stassfurter Chlorkaliumindustrie keinen daueraden Eingang verschaffen können. Auch die von Th. Schlösing*) beschriebene Methode, welche sich auf die Unlöslichkeit des überchlorsauren Kaliums stützt und deren Brauchbarkeit durch die Mittheilung von $\mathrm{Kraut}^{* *}$ ) nach den Versuchen von Orrmann und Küsel erwiesen ist, ist meines Wissens in Stassfurt nicht in Gebrauch gekommen. Seit längerer Zeit nit Kalibestimmungen beschäftigt, war ich bemüht alle die bei der Analyse etwa auftretenden Fehlerquellen zu ermittein und denjenigen analytischen Gang zu erforschen, welcher am schnellsten zum Ziele führt.

In den folgenden 3 Abtheilungen:

»I. die Darstellung des Platinchlorids,

Ir. die Löslichkeit der Platinchloriddoppelsalze der Alkalien und alkalischen Erden in Alkohol,

III. Operationen bei den Kalibestimmungen, «

gebe ich eine Uebersicht der in dieser Richtung angestellten Versuche. Eine Beschreibung der Darstellung des Platinchlorids dürfte deshalb nicht überflüssig erscheinen, weil von dessen Reinheit die Richtigkeit der Analyse wesentlich abhängt.

\section{Die Darstellung des Platinchlorids.}

In den meisten Fällen wird Platinchlorid aus den bei der Analyse gesammelten Platinsalzen hergestellt, welche vorzugsweise aus Kalium-

*) Compt rend. 73, 1269; diese Zeitschrift 11, 793.

**) Diese Zeitschrift 14, 152.

Fresenius, Zeitschrift. XVIII. Jahrgang. 
platinchlorid und aus der alkoholischen Waschflüssigkeit bestehen. Wird die alkoholische Lösung eingedampft, so bildet sich durch die Gegenwart des freien Platinchlorids das von $\mathrm{Z}$ eis $\mathrm{e}^{*}$ ) dargestellte entzündliche Platinchlorid $\mathrm{E}_{2} \mathrm{H}_{4}$ tt $\mathrm{Cl}_{2}$, welches durch Chlorkalium und Salmiak uicht mehr gefällt wird. Ausserdem scheidet sich auch ein im trocknen Zustande explosives Pulver (verpuffender Platinabsatz) ab, dessen Menge um so grösser ist, je mehr Platinchlorür in der Flüssigkeit vorhanden war, and welches hauptsächlich von der Einwirkung des Platinchlorürs auf Alkohol herrührt.

Das Reduciren der eingedampften Platinlösung kann durch verschiedene Mittel bewirkt werden; Natronlauge mit verschiedenen organischen Substanzen, wie Glycerin, Ameisensäure, Traubenzucker u. s. w. führen sehr schnell zum Ziele. In dem vorliegenden Falle dürite besonders Natriumcarbonat zu empfehlen sein, da auf die im Sieden befindliche alkoholische Platinlösung auch dieses rasch einwirkt. Kaliumplatinchlorid wird für sich allein durch Kochen mit Natriumcarbonat nicht verändert, trägt man es aber in dic obige, im Reduciren befindliche Flüssigkeit nach und nach ein, so wird es bei einer geniigenden Menge Soda ebenfalls vollständig reducirt. Das reducirte Platin (Platinmohr), welches ein leicht sich absetzendes, schwarzes Pulver darstellt, wird in der Porzellanschale durch Decantiren mit Salzsäure und Wasser ausgewaschen, bis es vollkommen frei von Schwefelsäure und Kalisalzen ist. Man verwende zum Reduciren nie Kalilauge, weil dadurch der Niederschlag stark kalihaltig und das Auswaschen erschwert wird. Soll Kaliumplatinchlorid für sich allein reducirt werden, so empfehle ich Natronlauge von 1,2 spec. Gew. mit 8 Proc. Glycerin. Schon in mässiger Wärme beginnt die Reduction und ist nach kurzem Sieden der Flüssigkeit vollständig beendet. Der aus alkoholischer Lösung reducirte Platinmohr enthält unter verschiedenen Umständen mehr oder weniger von dem oben erwahnten, verpuffenden Platinabsatz, welcher, auf dem Wasserbade getrocknet, verglimmt. Vollständig frei von diesem Körper wird das Platin jedoch nur in der Glühhitze; man versäume das Glühen nie, weil die kohlenstoffhaltige Platinverbindung in Königswasser schwer löslich ist, und weil andererseits die Platinlösung sofort durch fremde Stoffe verunreinigt wird. In dem aus Kaliumplatinchlorid mit Natronlauge und Glycerin reducirten Platinmohr habe ich einen verglimmenden

*) Poggendorff's Ann. 21, 498; 40, 249. 
Körper nicht wahrgenommen. Der auf diese Weise dargestellte reine Platinschwamm löst sich leicht in Salpetersalzsäure; ein unlöslicher Rückstand kann aus einer zufälligen Beimengung von Sand und Baryumsulfat oder aus Iridium bestehen, welches in geglühtem Zustande unlöslich ist.

Bei der Darstellung der Platinlösung sind verschiedene Vorsichtsmaassregeln zu beobachten, welche ich hier nur andeutungsweise wiedergeben kann. Man digerire bei mässiger Wärme mit einem Ueberschuss von Salzsäure und füge nach und nach Salpetersäure hinzu. Das Eindampfen muss bei gelinder Wärme vorgenommen werden, um der Bilaung von Platinchlorür möglichst vorzubeugen. Die stark concentrirte Platinlösung enthält stets Chlorür, welches bei niedrigerer Temperatur auf dem Wasserbade durch Zusatz von rauchender Salzsäure und wenig Salpetersäure in Chlorid äbergeführt wird. Ein etwa vorhandener Ueberschuss von Salpetersäure muss durch abwechselnden Zusatz von Salzsäure und Wasser fortgeschafit werden, weil durch dessen Gegenwart die Bildung von Stickoxydplatinchlorid bedingt und die Krystallisation des Platinchlorids befördert wird.

Stickoxydplatinchlorid $2 \mathrm{NO} \mathrm{Cl}$, $\mathrm{pt}_{4}$ bildet ein braungelbes bis pomeranzengelbes Krystallpulver, meistens aus Würfeln bestehend; es ist sehr zerfliessiich und äusserst leicht in Wasser löslich unter Entwicklung von Stickoxyd. Dabei zersetzt es sich nach folgender Gleichung: $2 \mathrm{~N} \theta \mathrm{Cl}$, et $\mathrm{Cl}_{4}+\mathrm{H}_{2} \theta=2 \mathrm{HCl}, \mathrm{PtCl}_{4}+\mathrm{N}_{2} \mathrm{O}_{3}$.

Die salpetrige Säure zersetzt sich aber sogleich mit dem Wasser in Stickoxyd und Salpetersäure; letztere kann nur durch Wiederholung der Operation aus dem Platinchlorid entfernt werden. Die beim Verdimnen mit Wasser ans dem Stickoxyd an der Luft sich bildende salpetrige Säure gibt ein scharfes Frkennungsmittel für die Gegenwart von Stickoxydplatinchlorid. Eine gut bereitete Platinlösung soll möglichst frei von Salpetersäure, Salzsäure und Platinchlorür sein. Die Herstellung einer solchen gelingt jedoch nur bei sorgfältiger und zeitraubender Arbeit. Die Gegenwart von Platinchlorür schadet nach meinen Erfahrungen mehr als die von Salpetersäure. Um den Einfluss der letzteren festzustellen, habe ich bei der Analyse wiederholt zu der Platinlösung 4 Proc. conc. Salpetersäure hinzugefügt, wie gewöhulich eingedampft und ein nur um 0,05 bis 0,1 Proc. zu niedriges Resultat erhalten, herrührend von der grösseren Löslichkeit des Kaliumplatinchlorids. Platinchlorür, welches ein in Wasser unlösliches, grüngelbes Pulver darstellt, ist in conc. 
512 Precht: Die Bestimmung d es Kaliums als Kaliumplatinchlorid.

Platinchloridlösung mit brauner Farbe löslich. Aus derselben scheidet es sich beim Verdünnen mit Wasser oder Alkohol theilweise aus, dagegen vollständig, wenn das Platinchlorid mit Chloralkalien gesättigt wird; es verursacht deshalb bei der Analyse zu hohe Resultate.

Verwendet man zur Darstellung der Platinlösung alte Platingeräthe, so wird das in denselben enthaltene Iridium ebenfalls gelöst, während das nicht legirte and geglühte Iridium in Königswasser unlöslich ist. Die Platinlösung bekommt durch einen Iridiumgehalt eine braune Farbe und das Ansehen, als sei Platinchlorür in derselben gelöst. Um eine vollständige Trennung des Iridiums vom Platin herbeizufähren, kann man unter anderem die von Gibbs entdeckte Trennungsmethode mittelst salpetrigsauren Kaliums anwenden. Obgleich nach den neuesten Untersuchungen von Seubert*) die Zahl 193 als Atomgewicht des Iridiums angegeben ist, so ist doch diese Abweichung von dem Atomgewichte des Platins zu gering, um bei der Kalibestimmung mittelst einer wenig iridiumhaltigen Platinlösung merkliche Differenzen zu verursachen. Ausserdem zeigt sich, dass durch Chlorkalium zunächst Platin gefällt wird und dann Iridium, denn das letztere findet sich vorzugsweise im alkoholischen Filtrat. Das zum Gebrauch bestimmte Platinchlorid muss sich vollständig in Alkohol lösen, und mit reinem Chlorkalium das berechnete Gewicht an Kaliumplatinchlorid ergeben.

Bei der Analyse ist es wünschenswerth, den Gehalt einer PlatinIösung annähernd zu kennen, ich füge deshalb hier eine Tabelle bei, welche den Gehalt an Platinchlorid in Procenten bei nebenstehendem spec. Gew. ergibt.

\begin{tabular}{|c|c|}
\hline $\begin{array}{l}\text { Proc. } \\
\text { Pt } \mathrm{Cl}_{4}\end{array}$ & $\begin{array}{l}\text { Proc. } \\
{\text { Pt } \mathrm{Cl}_{4}} \text { spec. Gew. }\end{array}$ \\
\hline $1=1,009$ & $10=1,097$ \\
\hline $2=1,018$ & $11=1,108$ \\
\hline $3=1,027$ & $12=1,119$ \\
\hline $4=1,036$ & $13=1,130$ \\
\hline $5=1,046$ & $14=1,141$ \\
\hline $6=1,056$ & $1 \breve{5}=1,153$ \\
\hline $7=1,066$ & $16=1,165$ \\
\hline $8=1,076$ & $17=1,176$ \\
\hline $9=1,086$ & $18=1,188$ \\
\hline
\end{tabular}

*) Ber. d. deutsch. chem. Gesellsch. z. Berlin 11, 1767. 
Proc.

Pt $\mathrm{Cl}_{4}$ spec. Gew.

$$
\begin{aligned}
& 19=1,201 \\
& 20=1,214 \\
& 21=1,227 \\
& 22=1,242 \\
& 23=1,256 \\
& 24=1,270 \\
& 25=1,285 \\
& 26=1,300 \\
& 27=1,315 \\
& 28=1,330 \\
& 29=1,346 \\
& 30=1,362 \\
& 31=1,378 \\
& 32=1,395 \\
& 33=1,413 \\
& 34=1,431
\end{aligned}
$$

Die zu den Bestimmungen benutzte Platinlösung enthielt auf 100 Th. Pt $\mathrm{tl}_{4} 2,24 \mathrm{Th}$. freie Salzsäure; die Bestimmung geschah in der Weise, dass eine gewogene Menge der Lösung auf dem Wasserbade eingedampft, im Wasserstoffstrom reducirt und das resultirende Platin gewogen wurde.

\section{Die Löslichkeit der Platinchlorid-Doppelsalze der Alkalien und alkalischen Erden in Alkohol.}

a. Kaliumplatinchlorid. Ueber die Löslichkeit des Kaliumplatinchlorids in Alkohol existirt bisher nur die Angabe von Fresenius*) nach welcher $1 \mathrm{Th}$. Salz $12083 \mathrm{Th}$. absoluten Alkohol, 3775 Th. Alkohol von 76 Proc. und 1053 Th. Alkohol von 55 Proc. zur Lösung bedarf. Nach diesen Zahlen würde es unbedingt nöthig sein, dem Löslichkeitsgrade und dem Quantum der Waschflüssigkeit entsprechend, für das analytische Resultat eine Correction zu berechnen, während solches in der Regel nicht geschieht. Um den Grad der Zuverlässigkeit der Angabe zu erkennen, bestimmte ich die Löslichkeit eines aus reinem Chlorkalium dargestellten und mit siedendem Wasser ausgewaschenen Kaliumplatinchlorids in Alkohol, indem ich $500 \mathrm{cc}$ der Flüssigkeit eindampfte und aus dem

*) Annal. der Chem. u. Pharm. 1846; 59, 117, - Fresenius, quant. Analyse, 6. Aufl. Bd. 1, p. 147. 
Rückstande das Platin reducirte. Als Mittel von je 3 Versuchen erhielt ich folgende Zahlen:

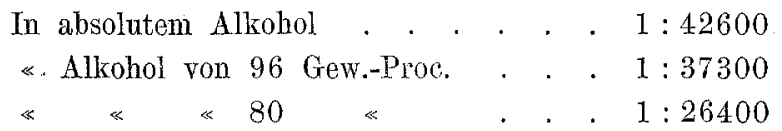

Diese Abweichung von Fresenius' Zahlen glaube ich auf einen, wenn auch nur geringen, Gehalt seiner Substanz an Kaliumplatinchlorür oder Chlorkalium zurückführen zu dürfen, auch mag sie daher rühren, dass Fresenius aus verhältnissmässig kleinen Mengen $(70-80 \mathrm{~g})$ der Lösung den Abdampfrückstand wog und somit auch die in dem Alkohol etwa vorhandenen Verunreinigungen. Um eine vollständig gesättigte Lösung zu erzielen, wurde der Alkohol mit dem Salze an dem Rade einer Maschine mehrere Tage geschüttelt und daher ist wohl die Annahme, dass meine Versuche mit einer ungesättigten Lösung vorgenommen sein könnten, ausgeschlossen.

b. Natriumplatinchlorid $2 \mathrm{NaCl}$, Pt $\mathrm{Cl}_{4}, 6 \mathrm{H}_{2} \ominus$. Aus einer concentrirten Lösung von Wasserstoffplatinchlorid und Chlornatrium kxystallisirt dieses Salz in Säulen und Tafeln des triklinischen Systems. Es lässt sich am leichtesten rein darstellen durch Lösen des trockenen. Salzes in heissem Alkohol. Das etwa im Ueberschuss vorhandene Chlornatrium bleibt ungelöst und Natriumplatinchlorid krystallisirt frei von Chlornatrium in grossen Krystallen mit 6 Molecülen Wasser. Mit dem so rein dargestellten Salze bestimmte ich die Löslichkeit in Wasser, Alkohol und Aether-Alkohol, welche bisher noch nicht bekannt war. Die angegebenen Zahlen beziehen sich auf wasserfreies $2 \mathrm{NaCl}, \pm \mathrm{Pt}_{4}$, berechnet aus dem reducirten Platin.

In kochendem Wasser löst sich das Salz fast in jedem Verhältnisse, eine wässerige, bei $15^{0} \mathrm{C}$. gesättigte Lösung von 1,368 spec. Gew. enthält 39,77 Proc. $2 \mathrm{NaCl}, \pm \mathrm{tCl}_{4}$ gelöst. Vermischt man die concentrirte Lösung mit einem gleichen Volum Alkohol, so scheidet sich das Salz in Krystallen aus, es ergibt sich also auch hier die bei vielen anderen Salzen beobachtete Thatsache, dass ein Gemisch von Alkohol und Wasser nicht so viel Salz zu lösen vermag, als das darin vorhandene Wasser. Eine gesättigte Lösung in Weingeist von 50 Gewichtsproc. enthält 17,85 Proc. Salz. Natriumplatinchlorid verliert auf dem Wasserbade bei einer Temperatur unter $100^{\circ}$ den grössten Theil des Krystallwassers. Das wasserfreie Salz löst sich in Allkohol unter Wärmeentwiclilung und in weit höherem Grade als das Salz mit 6 Molecülen $\mathrm{H}_{2} \theta$. Vollkommen 
bei $150^{\circ}$ entwässertes $2 \mathrm{NaCl}$, $\mathrm{PtCl}$ ' gab mit absolutem Alkohol eine Lösung, welche 48,3 Proc. Salz enthielt, und ein theilweise auf dem Wasserbade entwässertes Salz gab eine Lösung von 32,8 Proc. Fügt man zu solcher Lösung einige Tropfen Wasser, so erstarrt die Flüssigkeit únter Bildung des 6 fach gewässerten Salzes. Aus einer in der Wärme bereiteten Lösung des wasserfreien Salzes in Alkohol von 99,2 Gew.-Proc. schied sich beim Erkalten ein Gemenge des wasserfreien und des wasserhaltigen Salzes aus. Die Krystalle mit $6 \mathrm{H}_{2} \ominus$ lösen sich in absolutem Alkohol leichter als in 95 procentigem, wahrscheinlich herrührend von einem theilweisen Wasserverlust durch den stark hygroskopischen wasserfreien Alkohol. Die erste Lösung enthielt 11,90 Proc, die letztere 6,34 Proc. Die Löslichkeit des Natriumplatinchlorids, sowohl des wasserfreien als anch des wasserhaltigen, nimmt in der Wärme erheblich zu. Aus $200 \mathrm{~g}$ einer in der Wärme bereiteten Lösung in 90 procentigem Alkohol, welche bei $55^{0} \mathrm{C}$. zu krystallisiren begann, wurden $48 g$ Krystalle $=38,8 g 2 \mathrm{NaCl}$, $\mathrm{Et} \mathrm{Cl}_{4}$ gewonnen, oder - unter Berücksichtigung des spec. Gew. von 0,89 - enthielt die bei $55^{\circ} \mathrm{C}$. gesättigte Lösung 21,8 Proc. mehr gelöst als bei $15^{0} \mathrm{C}$. Die Mutterlauge enthielt 5,35 Proc., demnach waren in der Wärme 27,15 Proc. gelöst. Die bei $15^{0} \mathrm{C}$. gesättigte Lösung wird auf Zusatz von Aether gefällt und in einem Gemisch von gleichen Theilen Alkohol und Aether lösen sich 2,43 Proc. - In alkoholfreiem Aether ist das Salz unlöslich.

Natriumplatinchlorid wird durch Kochen mit Alkohol nicht verändert, kocht man dagegen eine mit Aether versetzte alkoholische Lösung, so zerfällt es theilweise in Chlornatrium und in lösliches Platinchlorid, welches dann in entzündliches Wasserstoffplatinchlorid übergegangen ist und durch Kalisalze nicht mehr gefällt wird.

c. Magnesiumplatinchlorid, $\mathrm{Ng} \mathrm{Cl}_{2}$, t. $\mathrm{Cl}_{4}, 6 \mathrm{H}_{2} \mathrm{\theta}$, stelte ich durch Zusammenfügen gleicher Molecüle $\mathrm{It} \mathrm{Cl}_{4}$ und $\mathrm{Mg} \mathrm{Cl}_{2}$ dar; das Salz lässt sich durch Krystallisation nicht reinigen, weil es nur schwierig in kleinen braungelben Krystallen krystallisirt. Die Krystalle mit $6 \mathrm{H}_{2} \theta$ gaben in absolutem Alkohol eine Lösung von 43,2 Proc. $M \mathrm{~g} \mathrm{Cl}_{2}, \mathbf{P t} \mathrm{Cl}_{4}$, und die bei $150^{\circ}$ getrockneten Krystalle eine Lösung yon 37,8 Proc. Es zeigt sich hier also nicht die beim Natriumplatinchlorid beobachtete Thatsache, dass das entwässerte Salz leichter löslich ist als das wasserhaltige.

d. Baryumplatinchlorid, $\mathrm{BaCl}_{2}, \mathrm{PtCl}_{4}, 8 \mathrm{H}_{2} \theta$. Dieses Salz bildet sich nor in einer lösung mit überschüssigem Chlorbaryum; gut 
516 Precht: Die Bestimmung des Kaliums als Kaliumplatinchlorid.

ausgebildete Krystalle erhält man durch freiwilliges Verdunsten neben conc. Schwefelsäure. Die Krystalle zerfallen in wässeriger Lösung theilweise, dagegen nahezu vollkommen durch Kochen mit absolutem Alkohol, in lösliches $\mathrm{Pt}_{4}$ und Chlorbaryum; letzteres ist in Alkohol unlöslich und lässt sich auf diese Weise bestimmen. Folgende Versuche wurden angestellt um den Grad der Zersetzung des Baryumplatinchlorids durch Alkohol festzustellen und um einen Maassstab fur den Einfluss des Chlorbaryums bei der Analyse zu erhalten. Eine Chlorbaryumlösung von bekanntem Gehalt wurde mit verschiedenen Mengen $\mathrm{PtCl}_{4}$ auf dem Wasserbade bis zur Krystallisation eingedampft, mit absolutem Alkohol ausgewaschen und das unlösliche $\mathrm{Ba} \mathrm{Cl}_{2}$ gewogen.

\begin{tabular}{|c|c|c|c|c|c|c|c|c|c|c|c|}
\hline \multicolumn{4}{|c|}{$\begin{array}{l}\text { Ein Molec. } \mathrm{Ba} \mathrm{Cl}_{2} \text { wurde } \\
\text { eingedampft mit } \pm t \mathrm{Cl}_{4} \\
\text { entsprechend: }\end{array}$} & & & & & \multicolumn{4}{|c|}{$\begin{array}{l}\text { Von dem vorh } \\
\mathrm{Ba} \mathrm{Cl}_{2} \text { waren als } \\
\text { ausgeschieden } \mathrm{i}\end{array}$} \\
\hline 11 & olec. & 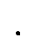 & & & & & & & . & & . $\quad 68,0$ \\
\hline 1,3 & « & $\cdot$ & • & & & & & & • & & - 61,3 \\
\hline 1,5 & « & . & . & & & & & & . & & . $\quad 53,4$ \\
\hline 1,8 & « & $\cdot$ & • & & $\therefore$ & & & $\cdot$ & - & & - $\quad 42,0$ \\
\hline 2,0 & $\ll$ & $\theta^{\circ}$ & . & & & & & & & & . $\quad 29,8$ \\
\hline 2,5 & « & $\cdot$ & - & & & & & • & - & & . 16,1 \\
\hline 3,0 & $\ll$ & $\theta^{\prime}$ & . & & & & & & - & & 5,2 \\
\hline 4,0 & « & . & & & . & . & & & . & - & 3,0 \\
\hline
\end{tabular}

Diese Versuche zeigen, dass selbst bei der 4 fachen Menge Platinchlorid noch nicht alles Chlorbaryum in einen in Alkohol löslichen Zustand übergeführt wird. Verschiedene Versuche für dasselbe Verhältniss von $\mathrm{Ba} \mathrm{Cl}{ }_{2}$ und $\mathrm{Pt} \mathrm{Cl}_{4}$ gaben verschiedene Zahlen, je nachdem die Lösung mehr oder weniger stark eingedampft wurde und grössere oder geringere Mengen freier Salzsäure enthielt; deshalb dürfen die angegebenen Zahlen nicht mit aller Strenge als richtig angesehen werden. Aus wasserfreiem Baryumplatinchlorid erhielt ich durch Auswaschen mit absolutem Alkohol 94,8 Proc. unlösliches Chlorbaryum.

f. Strontiumplatinchlorid bildet ein in Wasser ziemlich leicht lösliches Salz, welches sich mit absolutem Alkohol viel weniger zersetzt als das Baryumsalz. Die mit Alkohol vollständig ausgewaschenen Krystalle hinterlassen 4,2 Proc. unlösliches Chlorstrontium.

g. Calciumplatinchlorid, $\mathrm{CaCl}_{2}$, pt $\mathrm{Cl}_{4}, 8 \mathrm{H}_{2} \theta$, ist ein sehr leicht lösliches und an der Luft zerfliessendes Salz; die alkoholische Lösung enthielt 53 Proc. 


\section{Operationen bei den Kalibestimmungen.}

Bekanntlich stützt sich die Kalibestimmung auf die Unlöslichkeit des Kaliumplatinchlorids und auf die Löslichkeit des Natrium-, Magnesium- und Calciumplatinchlorids in Alkohol. Zur Bildung dieser Doppelsalze müssen sämmtliche Metalle als Chlorverbindungen zugegen sein, und soll durch Wägung des gefällten Kaliumplatinchlorids der Kaligehalt festgestellt werden, so dürfen auch keine Säuren vorhanden sein, welche mit den Alkalien in Alkohol unlösliche Salze bilden. Zur Ueberführung der neben Chlormetallen am häufigsten vorhandenen schwefelsauren Salze sind verschiedene Methoden ${ }^{*}$ ) vorgeschlagen, um die Anwendung von Chlorbaryum zu umgehen, weil das gefällte Baryumsulfat Alkalien mit niederreisst. Nach H. Rose werden die schwefelsauren Alkalien wiederholt bis zur Gewichtsconstanz mit reinem Salmiak geglüht, und L. Smith empfiehlt die Anwendung von Bleisalzen zur Fällung. In Stassfurt ist nur Chlorbaryum im Gebrauch und mit Recht, denn der dadurch entstehende Fehler ist so gering, dass derselbe bei den genauesten Analysen nicht in Frage kommt. Die Fällung wird bei $80-90^{\circ}$ in einer Lösung vorgenommen, welche auf 1 Theil Salz 0,5 Theil Salzsäure enthält, indem man nach und nach zu der häufig zum Sieden erhitzten Flüssigkeit soviel Chlorbaryum hinzufügt, dass die Schwefelsäure genau gefällt wird. Man erhält krystallinisches, sich schnell absetzendes Baryumsulfat und erkennt, wenn die Fällung in einer nicht zu kleinen Menge Flüssigkeit vorgenommen wird, (vortheilhaft in einem $\mathrm{zu}^{2 / 3}$ bis $3 / 4$ gefüllten Halbliterkolben) in der geklärten Flüssigkeit bei weiterem Zusatz von $\mathrm{Ba} \mathrm{Cl}_{2}$ sehr leicht die Endreaction. Ist mittelst Chlorbaryums und Schwefelsäure keine weitere Trübung zu erkennen, so wird der Inhalt abgekühlt und der Kolben bis zur Marke gefüllt. Ausserdem wird so viel Wasser nachgefüllt, als dem Volum des in der Flüssigkeit vorhandenen Baryumsulfats entspricht, welches sich aus dem spec. Gew. 4,2 und aus den gebrauchten Cubikcentimetern $\mathrm{Ba} \mathrm{Cl}_{2}$ ergibt. Die filtrirte Flüssigkeit ist zur Fällung des Kaliums fertig, man thut jedoch gut, sich von der Reinheit derselben zu überzeugen und sollte noch ejne geringe Menge Schwefelsäure zugegen sein, so kann dieselbe in dem Maassgefässe durch fein zerriebenes $\mathrm{Ba} \mathrm{Cl}_{2}$ gefällt werden; ein Ueberschuss von Chlorbaryum wird durch einige Tropfen Schwefelsäure beseitigt. Ich verwende stets eine Chlor-

*) Fresenius quantitative Analyse 6. Aufl. Bd. I p. 541. 
baryumlösung von bekanntem Gehalt, $104 g$ wasserfreies Salz im Liter, wodurch der Vortheil erreicht wird, dass zu den 'sich nahezu gleich bleibenden Producten der Kalisalzbergwerke - Kainit und Carnallit sofort die annähernd richtige Menge Chlorbaryum zugesetzt werden kann, und weil durch die dann folgende genaue Fällung gleichzeitig eine Schwefelsäurebestimmung erzielt wird. Um zu bestimmen, wie viel Alkalisalze dem auf diese Weise gefällten Baryumsulfat anhaften, wurde der Niederschlag mit siedendem Wasser ausgewaschen, getrocknet, geglüht und mit verdünnter Salzsäure gekocht. Die Salzsäure wurde abgedampft und der lösliche Rückstand bestimmt. $10 \mathrm{~g}$ Baryumsulfat gaben $22,3 \mathrm{mg}$ lösliche Salze, in welchen $9,1 \mathrm{mg}$ Chlorkalium vorhanden waren. Bei der Analyse des. Carnallits mit 12-14 Proc. Kieserit verursacht dieser Umstand einen Fehler von 0,045 Proc. $\mathrm{K} \mathrm{Cl}$ minus. Das Ausfällen der Schwefelsäure in neutraler. Lösung ist nicht zulässig, theils deshalb, weil in der trüben Lösung das Ende der Fällung nicht zu beobachten ist, aber namentlich deshalb, weil das aus neutraler Lösung gefällte Baryumsulfat viel Kaliumsulfat niederschlägt, wodurch Differenzen bis zu 1 Proc. hervorgerufen werden können. Das Ausfällen mit Chlorstrontium*) in alkoholischer Lösung statt mit Chlorbaryum kann ich nicht empfehlen.

Nach Finkener**) fällt man die Lösung von Kaliumsulfat und anderen in Wasser löslichen Salzen direct mit so viel Platinlösung als erforderlich ist, um alles Kalium in Kaliumplatinchlorid überzuführen, man dampft ein, wäscht mit Alkohol und sammelt den Niederschlag auf einem ungewogenen Filter. Das Kaliumplatinchlorid, welches mit anderen Salzen gemischt ist, wird reducirt, das Platin mit Wasser ausgewaschen und gewogen. Diese Methode gibt genaue Resultate und erfordert nur wenig Zeit mehr, als die Fällung der Schwefelsäure und Wägung des Kaliumplatinchlorids; sie ist namentlich dann zu empfehlen, wenn neben grossen Mengen Chlornatrium wenig Kalisaize vorhanden sind, weil dadurch viel Platinlösung und auch Alkohol erspart wird.

Wie allgemein bekannt, können bei den Kalibestimmungen neben Chlornatrium auch die Chlormetalle der alkalischen Erden zugegen sein, wenn so viel Platinlösung hinzugefügt wird, dass alle Chlorverbindungen in Platindoppelsalze übergeführt werden.

- Fresenius, quant. Analyse, 6. Aufl. Bd. I p. כ́41.

**) H. Rose's Handb. der analyt. Chem., 6. Aufl. v. R. Finkener 2, 923. 
Versuche von Souchay, *) welche auf Veranlassung von Fresenius angestellt wurden, ergaben bei Gegenwart von Chlormagnesium eine Gewichtszunahme von $0,6520 \mathrm{~g}$ Kaliumplatinchlorid um $0,0028 \mathrm{~g}$, 一 von Chlorbaryum um $0,0042 g$ - von Chlorstrontium um $0,0039 \mathrm{~g}$. Nach meinen Erfahrungen verursacht Chlormagnesium niemals eine Gewichtsvermehrung, weil Magnesiumplatinchlorid in Alkohol viel leichter löslich ist als Natriumplatinchlorid; dagegen erhält man bei Gegenwart von Chlorbaryum stets zu hohe Resultate, weil wie oben gezeigt wurde, das Baryumsalz in alkoholischer Lösung zerfällt. Diese Zersetzung findet bei Anwesenheit anderer in Alkohol löslicher Salze in erhöhtem Maasse statt, und das aus einer wenig Baryumsalze enthaltenden Lösung gefällte Kaliumplatinchlorid enthält stets Chlorbaryum als Verunreinigung. Die Versuche von Souchay sind jedenfalls mit einem grossen Ueberschuss von Platinchlorid ausgeführt, denn nur dadurch lässt sich der Zersetzung des Baryumplatinchlorids theilweise vorbeugen. Im allgemeinen gilt die Thatsache, dass eine Kalibestimmung nur dann richtig ausfällt, wenn die Lösung frei von Chlorbaryum**) und Schwefelsäure ist. Eine geringe Menge Schwefelsäure ist dann zulässig, wenn die Lösung nicht vollkommen zur Trockne verdampft wird. Zur Fällung mittelst Platinchlorids verwendet man 0,2 bis $1 \mathrm{~g}$ Substanz, je nach dem Gehalt an Chlorkalium. Für reine Kalisalze halte ich es für vortheilhaft $15,2805 \mathrm{~g}^{* * *}$ ) in $1 / 2$ Liter zu lösen und $10 \mathrm{cc}$ zu fällen, jedes Milligramm Kaliumplatinchlorid entspricht dann 0,1 Proc. Chlorkalium and eine Rechnung ist somit nicht erforderlich. Wird der Kaligehalt als Kaliumsulfat angegeben, so werden $17,8470 \mathrm{~g}$ Substanz gelöst und weiter wie oben verfahren, jedes Milligramm Kaliumplatinchlorid zeigt dann 0,1 Proc. Kaliumsulfat an. Um eine grössere Genauigkeit zu erzielen, ist zur Analyse des in den Bergwerken gewonnenen Carnallits, welcher durchschnittlich 16 Proc. K Cl enthält, eine grössere Menge Substanz wünschenswerth (vortheilhaft $1 g$ in $25 c c$ einer Lösung von $20 \mathrm{~g}$ in $1 / 2 l$ ). Die mit genügendem Platinchlorid versetzte Lösung wird auf dem Wasserbade so weit eingedampft, dass beim Erkalten die syrupdicke Flüssigkeit schnell in feinen Krystallen anschiesst. Es ist von Wichtig-

*) Diese Zeitschrift 16, 65 .

**) Vergl. jedoch hierzu den Versuch Souchay's a. a. O. S. 67. R. F.

***) Die Wägung geschieht am bequemsten auf einer kleinen Handwage, wozu man sich ein bestimmtes Gewicht für Chlorkalium und ein anderes für Kaliumsulfat von der angegebenen Schwere herstellen lässt. Ich habe solche Gewichte von dem Mechaniker Westphal in Celle für 1 M. 50 Pf. erhalten. 
keit, darauf zu achten, dass in der eingedampften Lösung sich nie grössere Krystalle von Natriumplatinchlorid bilden, weil dadurch das Auswaschen sehr erschwert wird. Ist neben Kaliumplatinchlorid nur Natriumplatinchlorid vorhanden, so kann man mit Vortheil die Lösung vollständig bis zur Trockne verdampfen, um das Natriumplatinchlorid zu entwässern, wodurch eine grössere Löslichkeit desselben in absolutem Alkohol bedingt wird. Das Auswaschen geschieht in der Porzellanschale durch Decantiren mit absolutem Alkohol, indem der Abdampfrückstand mit einem gebogenen Glasstabe fein zerrieben wird. Die aus der Porzellanschale abgegossene Flüssigkeit wird durch ein gewogenes Filter filtrirt und wenn das Auswaschen beendet ist, das Kaliumplatinchlorid auf das Filter gebracht. Durch Reiben mit dem gebogenen Glasstabe entfernt man die letzte Spur Kaliumplatinchlorid von der Wandung der Porzellanschale. Das Filtriren geschieht stets mit Hülfe eines Saugapparates. Die zuerst aufgegossene Flüssigkeit, welche viel Platinsalze gelöst enthält, darf den Rand des Filters nicht berühren, weil dadurch das Auswaschen erschwert wird, was sich beim Trocknen des Filters durch einen gefärbten Rand zu erkennen gibt. In der Regel kann man annehmen, dass ein vollständiges Auswaschen mit 60-70 cc Alkohol in 10 Minuten erreicht wird. Eine Mischung von Alkohol und Aether, welche meistens zum Auswaschen empfohlen wird, halte ich für unvortheilhaft, weil Natriumplatinchlorid in derselben sehr schwer löslich ist. Wie schon oben erwähnt, ist Natriumplatinchlorid in heissem Alkohol viel leichter löslich, deshalb ist es vortheilhaft, wenn man viel zu filtriren hat, mit heissem Alkohol auszuwaschen; eine Reduction des Platinchlorids findet dabei nicht statt.

Das Wägen des Kaliumplatinchlorids auf einem gewogenen Filter gibt genaue Resultate, wenn das Filter vor dem Gebrauch genügend lange (etwa 2 Stunden) bei $130^{\circ} \mathrm{C}$. getrocknet wird. Das Filter mit dem Niederschlage, welches mit absolutem Alkohol ausgewaschen ist, wird bei $130^{\circ} \mathrm{C}$. in 15 bis 20 Minuten vollkommen trocken und eine spätere Wägung ergibt keine Gewichtsabnahme. Das vielfach empfohlene Asbestfilter ist weniger vortheilhaft und in Stassfurt meines Wissens nicht in Gebrauch gekommen.*)

Um eine grössere Löslichkeit des Natriumplatinchlorids zu erzielen,

*) Dass man die Wägung eines jeden Filters leicht ersparen kann, habe ich in dieser Zeitschr. 16, 64 mitgetheilt. R. F. 
wurde von Ulex*) der Zusatz von Glycerin empfohlen. Glycerin ist jedenfalls nicht nachtheilig, aber ein wesentlicher Vortheil wird durch dessen Anwendung nicht erreicht, weil sich, wenn man die Lösung stark eindampft, ein zäher Rückstand bildet, welcher sich schwer auswaschen lässt.

Hat man geringe Mengen Chlorkalium neben viel Chlornatrium zu bestimmen, wie es häufig bei Steinsalzanalysen vorkommt, so dampft man eine grössere Quantität Salz $(10-100 \mathrm{~g})$ mit einer Natriumplatinchloridlösung von bekanntem Gehalt ein, wodurch die Kalisalze gefällt werden. Das im Ueberschuss hinzugefügte Natriumplatinchlorid wird mit absolutem Alkohol ausgewaschen und im Filtrat wird das Platin durch Reduction abgeschieden, ausgewaschen und gewogen. Diese Methode ist besonders zu empfehlen für die Analyse von Salzen, welche weniger als 2 Proc. Chlorkalium enthalten, sie ist dann die einzige, welche richtige Resultate liefert.

Neu Stassfurt bei Stassfurt, April 1879.

\section{Maassanalytische Bestimmung der Schwefelsänre in schwefel- sauren Salzen.}

Von

\section{Dr. H. Precht.}

Von den verschiedenen Methoden zur maassanalytischen Bestimmang. der Schwefelsäure in schwefelsauren Salzen ist die Methode der Fällung durch Chlorbaryum der'allgemeinsten Anwendung fähig und lässt sich nach einiger. Uebung auch schnell and mit genügender Genauigkeit ausführen.

Zum Titriren verwende ich eine Normal-Chlorbaryumlösung (104 g wasserfreies Salz im Liter), welche vortheilhaft in einer Flasche der Art aufbewahrt wird, dass die Lösung in die dazu bestimmte Bürette atis der hochstehenden Flasche von unten einfliesst. Die Chlorbaryumlösung bedecke ich in der verschlossenen Flasche mit einer $1 \mathrm{~cm}$ hohen Schicht Petroleumäther und lasse, wenn die leichtflüchtigen Kohlenwasserstoffe nicht mehr die zum Ausfliessen der Flüssigkeit erforderliche Tension besitzen, durch Oeffnen eines Glasrohrs mit Quetschhahn Luft einströmen; auf diese Weise ist die Lösung vor Verdunstung geschützt. Wenn die Lösung der schwefelsauren Salze klar ist, lässt sich die End-

*) Naturf.-Vers. 1877 p. 144; diese Zeitsehr. 17, 175. 